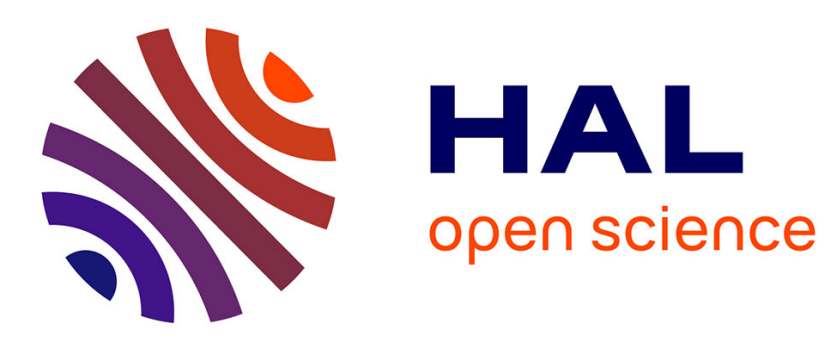

\title{
Roton collective mode observed in a two-dimensional Fermi liquid
}

Henri Godfrin, Matthias Meschke, Hans-Jochen Lauter, Ahmad Sultan, Helga M. Böhm, Eckhard Krotscheck, Martin Panholzer

\section{- To cite this version:}

Henri Godfrin, Matthias Meschke, Hans-Jochen Lauter, Ahmad Sultan, Helga M. Böhm, et al.. Roton collective mode observed in a two-dimensional Fermi liquid. Nature, 2012, 483, pp.576. 10.1038/nature10919 . hal-00920422

\section{HAL Id: hal-00920422 \\ https://hal.science/hal-00920422}

Submitted on 20 Dec 2013

HAL is a multi-disciplinary open access archive for the deposit and dissemination of scientific research documents, whether they are published or not. The documents may come from teaching and research institutions in France or abroad, or from public or private research centers.
L'archive ouverte pluridisciplinaire HAL, est destinée au dépôt et à la diffusion de documents scientifiques de niveau recherche, publiés ou non, émanant des établissements d'enseignement et de recherche français ou étrangers, des laboratoires publics ou privés. 


\title{
Roton collective mode observed in a two-dimensional Fermi liquid
}

\author{
Henri Godfrin $^{1}$, Matthias Meschke ${ }^{1,2}$, Hans-Jochen Lauter ${ }^{3,4}$, Ahmad Sultan ${ }^{1}$, \\ Helga M. Böhm ${ }^{5}$, Eckhard Krotscheck ${ }^{5,6}$ \& Martin Panholzer ${ }^{5}$
}

${ }^{1}$ Institut Néel, CNRS et Université Joseph Fourier, BP 166, 38042 Grenoble Cedex 9, France

${ }^{2}$ Low Temperature Laboratory, Aalto University, PO Box 15100, 00076 Aalto, Finland

${ }^{3}$ Institut Laue-Langevin, BP 156, 38042 Grenoble Cedex 9, France

${ }^{4}$ Oak Ridge National Laboratory, PO Box 2008, Oak Ridge TN 37831-6475, USA

${ }^{5}$ Institute for Theoretical Physics, Johannes Kepler University, A-4040 Linz, Austria

${ }^{6}$ Department of Physics, University at Buffalo SUNY, Buffalo, NY,14260, USA 
Understanding the dynamics of correlated many-body quantum systems has been a challenge for modern physics. Due to the simplicity of their Hamiltonian, ${ }^{4} \mathrm{He}$ (bosons) and ${ }^{3} \mathrm{He}$ (fermions) have served as paradigm for strongly interacting quantum fluids. For this reason, substantial efforts have been devoted to their understanding. An important milestone was the direct observation of the collective "phonon-roton" mode in liquid ${ }^{4} \mathrm{He}$ by neutron scattering, verifying Landau's prediction $^{1}$ and his fruitful concept of elementary excitations.

In a Fermi system, collective density fluctuations ("zero-sound" in ${ }^{3} \mathrm{He}$, "plasmons" in charged systems) as well as incoherent particle-hole (PH) excitations are observed. At small wave-vectors and energies, both types of excitations are described by Landau's theory of Fermi liquids ${ }^{2,3}$. At higher wavevectors, the collective mode enters the PH band, where it is strongly damped. The dynamics of Fermi liquids at high wave-vectors was thus believed to be essentially incoherent.

We report here the first observation of a roton-like excitation in a Fermi liquid, obtained in a monolayer of liquid ${ }^{3} \mathrm{He}$, studied by inelastic neutron scattering. We find that the collective density mode reappears as a well-defined excitation at momentum transfers larger than twice the Fermi momentum. We thus observe unexpected collective behaviour of a Fermi many-body system in the region outside the scope of Landau's theory. A satisfactory interpretation of the measured spectra is obtained within a novel dynamic many-body theory ${ }^{4}$.

Quantum many-body systems are ubiquitous in nature. The identification of their ground state and the description of their elementary excitations is a cornerstone of modern Physics. Nuclei, metals, semiconductors, and neutron star matter, are examples of quantum fluids. Their properties depend, among others, on the quantum statistics obeyed by the particles (electrons, nucleons, atoms), leading to the classification in terms of Bose or Fermi systems. Weakly interacting Bose and Fermi systems are well 
understood, however, the road from these gases to their strongly interacting analogue was not smoothly paved, and considerable attention has been devoted to correlated quantum systems ${ }^{1-6}$. The interplay between statistics and interactions in quantum manybody systems is the central theme of this Letter.

Our work is concerned with the helium liquids, the canonical quantum fluids. ${ }^{4} \mathrm{He}$ is a boson, whereas, due to its nuclear spin $1 / 2,{ }^{3} \mathrm{He}$ is a fermion. At $2.17 \mathrm{~K}$, ${ }^{4} \mathrm{He}$ becomes superfluid. Liquid ${ }^{3} \mathrm{He}$, however, remains a normal Fermi liquid down to millikelvin temperatures, where Cooper pairs form and condense into several superfluid phases; it is the neutral analogue of the conduction electrons in a metal. Clearly, Bose and Fermi liquids behave differently, and are thus expected to sustain very different excitations.

The elementary excitations of a Bose fluid have been described by Landau ${ }^{1}$. Their dispersion relation (Figure1) shows a sharp linear "phonon" mode, which evolves continuously as a function of the wave-vector, displaying a pronounced "roton" minimum $^{1,6-9}$. The excitations remain well-defined even at atomic wave-vectors and at relatively high temperatures. Modern many-body theories have proven successful in describing the dynamics of Bose fluids under different conditions of density and dimensionality: bulk, films, or droplets ${ }^{10-12}$.

The low lying elementary excitations of liquid ${ }^{3} \mathrm{He}$ (Fig. 2) are incoherent particle-hole $(\mathrm{PH})$, as well as collective density and spin-density modes, described by Landau's Fermi liquid theory ${ }^{2,3,8}$. For intermediate energies, a qualitative description is provided by the Random Phase Approximation (RPA), ${ }^{3,5,8}$. In the RPA, PH states are confined (Figure 2) within the particle-hole band (PHB). The boundaries of the PHB for a noninteracting system (the Fermi "gas") are $E_{\mathrm{max}, \min } / E_{\mathrm{F}}=\left(k / k_{\mathrm{F}}\right)^{2} \pm 2\left(k / k_{\mathrm{F}}\right)$ where $E_{\mathrm{F}}=$ $\hbar^{2} k_{\mathrm{F}}^{2} / 2 m$ is the Fermi energy, $k$ the excitation wave-vector, $k_{\mathrm{F}}$ the Fermi wave-vector, and $m$ the (bare) mass of a particle.

Landau's Fermi Liquid theory postulates that an interacting system behaves like a Fermi gas with renormalized parameters. In particular, an effective mass $m *$ is assigned 
to the fermionic "quasi-particles". The theory describes well the low temperature properties of bulk liquid ${ }^{3} \mathrm{He}$, where $m^{*}$, depending on the liquid pressure, varies from 3 to 6 times $m$. This picture does not apply at high wave-vectors: it was theoretically shown ${ }^{13-15}$, and experimentally verified ${ }^{16}$ in bulk liquid ${ }^{3} \mathrm{He}$, that the effective mass enhancement is confined to the vicinity of the Fermi surface. Therefore, the PHB is expected to be essentially that calculated for the non-interacting system, except for very low energies, where it is strongly depressed.

The density collective mode, called zero-sound, is described by Landau as an oscillation of the whole Fermi sphere ${ }^{2,3,8}$. Contrarily to ordinary sound, its frequency is higher than the collision rate. First detected by ultrasonic techniques, it has been investigated in detail by neutron scattering ${ }^{16-18}$. Zero-sound (Fig. 2) has a linear dispersion relation above the PHB, then a negative deviation at intermediate wave-vectors, and finally enters the PHB, where this mode disappears due to decay into PH excitations (Landaudamping).

The corresponding excitation in electron fluids is the plasmon. Apart from an energy gap at zero wave-vector due to charge, the physics is the same ${ }^{3}$. In particular, the plasmon dispersion curve is observed to enter the PHB, and to disappear, as shown in Figure 2.

A very elegant discussion of the dynamics of Fermi many-body systems has been given in a review article by D. Pines ${ }^{6}$. He stated that the phonon-roton mode of liquid ${ }^{4} \mathrm{He}$ and the zero-sound mode of liquid ${ }^{3} \mathrm{He}$ have strong interactions, rather than quantum statistics, as common origin. Ph. Nozières ${ }^{9}$ argued that the physical origin of the roton minimum may be the incipient localisation of the particles due to interactions.

In the present experiment, we have determined the dynamic structure factor of a monolayer of liquid ${ }^{3} \mathrm{He}$, essentially at zero temperature. Two-dimensional Fermi liquids have been extensively investigated by thermodynamic techniques ${ }^{19-24}$; we present here the first direct investigation of their elementary excitations by neutron 
scattering. We observe a collective mode, which remains visible throughout the whole PHB, and re-emerges as a well-defined mode at large wave-vectors, as shown in Fig. 3. The helium film is made at low temperatures by the controlled adsorption of gas onto a substrate, a high quality ZYX exfoliated graphite (surface area $60 \mathrm{~m}^{2}$ ) with large coherence length $(190 \mathrm{~nm})$ and low mosaic spread $\left(10^{\circ}\right)$, essential for obtaining good resolution neutron spectra ${ }^{24,25}$. The substrate was first pre-plated by a complete monolayer of ${ }^{4} \mathrm{He}$. This high density solid provides a smoother adsorption potential than bare graphite. A monolayer of liquid ${ }^{3} \mathrm{He}$ is then deposited onto the ${ }^{4} \mathrm{He}$ pre-plated substrate. The amount of ${ }^{4} \mathrm{He}$ introduced in the cell was $\mathrm{V}_{4}=28.59 \mathrm{~cm}^{3}$ STP (volume of gas under standard conditions of temperature and pressure). This is sufficient to complete the first monolayer considering the effect of the pressure of the partial second layer. The amount of ${ }^{3} \mathrm{He}$ was $\mathrm{V}_{3}=11.0 \mathrm{~cm} 3 \mathrm{STP}$. Using a coverage scale developed earlier $^{24}$ (supplementary information is provided on-line), we obtain a lower limit $A_{c}=59.7 \mathrm{~m}^{2}$ and an upper limit $A_{I}=65.3 \mathrm{~m}^{2}$ for the area available for the ${ }^{3} \mathrm{He}$ layer adsorption; the liquid ${ }^{3} \mathrm{He}$ density is determined to be $\rho_{3}=4.7 \pm 0.2$ atoms $/ \mathrm{nm}^{2}$. At this areal density, the ${ }^{3} \mathrm{He}$ effective mass at the Fermi surface ${ }^{19-23}$ is $m * / m \sim 4$, similar to that of bulk liquid ${ }^{3} \mathrm{He}$ at a pressure of $1 \mathrm{MPa}$. An aluminium sample cell confines the gas during the adsorption process, performed through a filling capillary. Measurements are made in a dilution refrigerator, at temperatures below $100 \mathrm{mK}$.

The experiments were performed at the Institut Laue-Langevin on the time-of-flight spectrometer IN6, using incident wavelengths of 0.512 and $0.41 \mathrm{~nm}$. The measured dynamic structure factor $S(k, E)$ contains all the relevant information on the elementary excitations of a system; it gives the probability for creating an excitation with wavevector $k$ and energy $E$. The main features revealed by our data, shown in Figure 3, are: 1) the zero-sound mode is seen at low wave-vectors; given the limited experimental range, its definite identification requires theoretical support, to be presented later. It is found at energies well below those observed in bulk liquid ${ }^{3} \mathrm{He}$. 2) The mode is 
broadened as it enters the PHB. 3) It emerges beyond the limits of the PHB as an intense mode, displaying a minimum as a function of energy; its energy increases rapidly beyond this minimum. 4) The high intensity region of $S(k, E)$ closely resembles the phonon-maxon-roton dispersion relation of liquid ${ }^{4} \mathrm{He}$. 5) Significant intensity is present at low wave-vectors above the PHB, demonstrating the existence of multi-pair excitations.

We now show that dynamic many-body effects play an essential role in explaining the observed position of the roton and the emergence of the collective mode beyond the PHB. Adopting the view that the physical mechanisms that determine the shortwavelength spectrum are the same in ${ }^{4} \mathrm{He}$ and in ${ }^{3} \mathrm{He}$, we have developed the fermion generalisation $^{4}$ of the dynamic many-body theory of Jackson, Feenberg, and Campbell ${ }^{10,12}$. The boson theory has by now been brought to a level where a consistent description of the dynamics of ${ }^{4} \mathrm{He}$ in the whole $(k, E)$ plane is possible. The fermion version of the theory ${ }^{4}$ allows calculating the dynamics of strongly interacting systems in the language of a time-dependent Hartree-Fock theory ${ }^{5}$ with energy-dependent effective interactions. It supersedes the intuitive "backflow" picture of Feynman" and goes beyond the RPA by being applicable at atomic wave-vectors.

The results of our calculation of the dynamic structure factor at the density 4.9 atoms $/ \mathrm{nm}^{2}$ are shown in Figure 4. A good quantitative agreement with the experiment at a similar density is obtained, without any adjustable parameters. The most important findings are: 1) At low wave-vectors, the presence of a long-lived zero-sound collective mode, close to the PHB upper limit. 2) The mode is broadened, but visible, within the PHB. 3) It emerges from the PHB as a well-defined, intense excitation. 4) A phonon-maxon-roton type of dispersion relation is clearly seen. 5) Multi-pair excitations are present at low wave-vectors above the PHB, causing a natural width of the phonon. The contour plots of Fig. 4 show a strong down-shift in the density of states compared to the RPA predictions. This is more clearly seen in the spectra at constant wave-vector 
presented in Fig. 5. The experiments yield strongly asymmetric spectra, with a marked peak at low energy. RPA calculations display qualitatively different asymmetric spectra, the peak being at high energy. This effect was previously seen in bulk ${ }^{3} \mathrm{He}$ measurements and had impaired their analysis ${ }^{16}$. Our dynamic many-body calculation ${ }^{4}$ applied here gives a much better description of the spectra.

The measured spectra are broadened due to the finite experimental resolution (grouping detectors yields $\Delta k=1 \mathrm{~nm}^{-1}$ and the instrument geometry yields $\Delta E=0.1 \mathrm{meV}$ ). The dynamic many-body calculation convoluted with the experimental resolution provides calculated spectra (Fig. 4) whose widths agree with the measured ones. Therefore, the energy width of the collective mode observed here outside the particle-hole band is certainly smaller than or equal to the energy resolution. This should not be confused with the extremely sharp phonon-roton mode of liquid ${ }^{4} \mathrm{He}$ in the superfluid state: liquid ${ }^{3} \mathrm{He}$ sustains, in addition to the collective density mode, incoherent particle-hole excitations, which obviously open additional decay channels, see point 5 above.

A word is in order on our use of a non-interacting single-particle spectrum for determining the boundaries of the PHB. Incoherent particle-hole excitations are limited to a band $E(q-k)-E(k)<\hbar \omega<E(q+k)-E(k)$ where $k$ is inside the Fermi sea. The single particle spectrum $E(k)$ deviates from the free spectrum for two reasons: One is the corrugation of the substrate, leading to the so-called "band-mass" which is of the order $m_{\text {band }} \approx 1.2 m$ (L. Reatto, communication presented at the International Conference on Low Temperature Physics, LT26, Beijing, August 2011). Second, there is an interaction contribution. It exhibits a peak at the Fermi wave-vector $k_{F}$, but drops rapidly as a function of momentum in both three and two dimensions ${ }^{13-16}$. As a consequence, the particle-hole band is modified only up to about the Fermi energy by interaction and band-structure effects.

To summarise, we have observed for the first time the elementary excitations of twodimensional liquid ${ }^{3} \mathrm{He}$ in a large energy/wave-vector range. Using the favourable 
conditions displayed by this system, we have demonstrated that a strongly interacting quantum many-body system sustains collective density excitations which are largely independent from the quantum statistics: the fermionic ${ }^{3} \mathrm{He}$ collective mode has the same physical origin as the phonon-roton curve of the bosonic ${ }^{4} \mathrm{He}$. To achieve a theoretical understanding of these phenomena, a dynamical treatment of short range correlations has been successfully implemented.

Generalising Ruvalds's proposal ${ }^{26}$ of a superconducting pairing mechanism mediated by long wavelength plasmons, our observation of a roton-like coherent mode characterised by a high density of states leads us to suggest a novel pairing mechanism, mediated by high-momentum density fluctuations. The consequences of the presence of plasmon collective modes at high wave-vectors on the dynamics of electronic systems ${ }^{27-30}$ like high- $T_{c}$ superconductors, heavy fermions, metals, graphene, and others, definitely deserve exploration. 


\section{References}

1. Landau, L.D. On the theory of superfluidity of helium II. J. of Phys. Moscow 11, 91 (1947).

2. Landau, L.D. The theory of a Fermi liquid. Soviet Physics JETP 3, 920 (1957).

3. Pines, D. \& Nozières, P. The Theory of Quantum Liquids. Benjamin, New York (1966).

4. Böhm, H. M., Holler, R., Krotscheck, E. \& Panholzer, M. Dynamic many-body theory: Dynamics of Strongly Correlated Fermi Fluids. Phys. Rev. B 22, 224505 (2010).

5. Thouless, D.J. The Quantum Mechanics of Many-body Systems. 2nd ed. Academic Press, New York (1972).

6. Pines, D. Elementary excitations in quantum liquids. Physics Today 34, 106-131 (Nov. 1981).

7. Feynman, R.P. \& Cohen, M. Energy spectrum of the excitations in liquid helium. Phys. Rev. 102, 1189-1204 (1956).

8. Glyde, H.R. Excitations in Liquid and Solid Helium. Clarendon Press, Oxford (1994).

9. Nozières, P. Is the roton in superfluid ${ }^{4} \mathrm{He}$ the ghost of a Bragg spot? J. of Low. Temp. Phys. 137, 45-67 (2004).

10. Jackson, H. W. Perturbative form of $S(k, \omega)$ for liquid ${ }^{4} \mathrm{He}$ : basic calculation and results. Phys. Rev. A 8, 1529-1535 (1973).

11. Clements, B.E., Krotscheck E. \& Tymczak, C.J. Multiphonon excitations in boson quantum films. Phys. Rev. B 53, 12253-12275 (1996).

12. Campbell, C.E. \& Krotscheck, E. Dynamic many-body theory: pair fluctuations in bulk ${ }^{4}$ He. Phys. Rev. B. B 80, 174501 (2009).

13. Friman, B.L. \& Krotscheck, E.K. Zero sound, spin fluctuations, and effective mass in liquid ${ }^{3}$ He. Phys. Rev. Lett. 49, 1705-1708 (1982).

14. Krotscheck, E. \& Springer, J. Physical mechanisms for effective mass enhancement in ${ }^{3}$ He. J. of Low Temp. Phys. 132, 281-295 (2003). 
15. Boronat, J., Casulleras, J., Grau, V. Krotscheck, E. \& Springer, J. Effective mass of two-dimensional ${ }^{3}$ He. Phys. Rev. Lett. 91, 085302 (2003).

16. Glyde, H.R., Fak, B., van Dijk, N.H., Godfrin, H., Guckelsberger K. \& Scherm, R. Effective mass, spin fluctuations and zero sound in liquid ${ }^{3}$ He. Phys. Rev. B 61, 1421$1432(2000)$.

17. Sköld, K., Pelizzari, C.A., Kleb, R. \& Ostrowski, G.E. Neutron scattering study of elementary excitations in liquid helium-3. Phys. Rev. Lett. 37, 842-845 (1976).

18. Scherm, R., Gluckelsberger, K., Fak, B., Sköld, K., Dianoux, A.J., Godfrin, H. \& Stirling, W.G. Pressure dependence of elementary excitations in normal liquid helium-3. Phys. Rev. Lett. 59, 217-220 (1987).

19. Greywall, D.S. Heat capacity of multilayers of ${ }^{3} \mathrm{He}$ adsorbed on graphite at low millikelvin temperatures. Phys. Rev. B 41, 1842-1862 (1990).

20. Morhard, K.D., Bäuerle, C., Bossy, J., Bunkov, Yu.M., Fisher, S.N. \& Godfrin, H. Two-dimensional Fermi liquid in the highly correlated regime: the second layer of ${ }^{3} \mathrm{He}$ adsorbed on graphite. Phys. Rev. B 53, 2658-2661 (1996).

21. Bäuerle, C., Bunkov, Yu.M., Chen, A.S., Fisher, S.N. \& Godfrin, H. Ultra-low temperature magnetic properties of liquid ${ }^{3} \mathrm{He}$ films. J. of Low Temp. Phys. 110, 333338 (1998).

22. Casey, A., Patel, H., Nyéki, J., Cowan, B.P. \& Saunders, J. Strongly correlated two dimensional fluid ${ }^{3}$ He. J. of Low Temp. Phys. 113, 293-298 (1998).

23. Neumann, M., Nyéki, J., Cowan, B.P. \& Saunders, J. Bilayer ${ }^{3}$ He: a simple twodimensional heavy-fermion system with quantum criticality. Science 317, 1356-1359 (2007).

24. Godfrin H. \& Lauter, H.J. Progress in Low Temp. Physics, Vol.XIV, Chapter 4, p.213-320, ed. W.P. Halperin, Elsevier Science B.V., Amsterdam (1995).

25. Lauter, H.J., Godfrin, H., Frank, V.L.P. \& Leiderer, P. Ripplons in ${ }^{4} \mathrm{He}$ films observed by neutron scattering. Phys. Rev. Lett. 68, 2484-2487 (1992). 
26. Ruvalds, J. Are plasmons the key to superconducting oxides? Nature 328, 299-299 (1987).

27. Bostwick, A., Ohta, T., Seyller, T., Horn, K. \& Rotenberg, E. Quasiparticle dynamics in graphene. Nature Physics 3, 36-40 (2007).

28. Diaconescu et al. Low-energy acoustic plasmons at metal surfaces. Nature 448, 57 59 (2007).

29. Uemura, Y.J. et al. Phase separation and suppression of critical dynamics at quantum phase transitions of $\mathrm{MnSi}$ and $\left(\mathrm{Sr}_{1-\mathrm{x}} \mathrm{Ca}_{\mathrm{x}}\right) \mathrm{RuO}_{3}$, Nature Physics 3, 29-35 (2007). 30. Kohsaka, Y. et al. How Cooper pairs vanish approaching the Mott insulator in $\mathrm{Bi}_{2} \mathrm{Sr}_{2} \mathrm{CaCu}_{2} \mathrm{O}_{8+\delta}$, Nature 454, 1072-1078 (2008). 
Supplementary Information is linked to the online version of the paper at www.nature.com/nature

Acknowledgements We are grateful to the Austrian-French programme "Amadeus" for providing initial support, to the Austrian Science and the French ANR (project High-Q Fermions) and the EU FRP7 low temperature infrastructure grant "Microkelvin" for funding this research, and the Institut Laue-Langevin for the use of the facility.

Author contributions H. G, M. M, H.-J. L., A. S. and M. P. performed the neutron experiments; H. B., E. K. and M. P. developed the theory. All authors analysed the results and contributed to the work.

\section{Author information}

Reprints and permissions information is available at npg.nature.com/reprints. The authors declare no competing financial interests.

Correspondence and requests for materials should be addressed to H.G. (henri.godfrin@grenoble.cnrs.fr) 


\section{Figure legends}

Figure 1 Elementary excitations of superfluid ${ }^{4} \mathrm{He}$. The solid line is the dispersion relation predicted by L.D. Landau ${ }^{1}$. Crosses correspond to the excitation energy as a function of wavevector determined by neutron scattering (see ref. 8). At low wave-vectors, the dispersion relation is linear, and the excitations are quantised sound waves (phonons). At higher wavevectors, the spectrum evolves continuously, displaying a maximum and then a characteristic minimum. The corresponding excitations are called respectively, maxons and rotons; the latter play an essential role in the thermodynamic properties of superfluid ${ }^{4} \mathrm{He}$.

Figure 2 Schematic picture of the elementary excitations of a Fermi liquid. The broad shaded area corresponds to the particle-hole band, i.e., to the excitation energy range as a function of wave-vector accessible by promoting a particle occupying a state inside the Fermi surface, to an empty state outside it. In addition, an interacting Fermi system displays collective density modes, called "plasmons" in charged, and "zero-sound" in neutral systems. With increasing wave-vectors, the collective modes enter the particle-hole band, where they decay (Landau damping) into incoherent particle-hole excitations.

Figure 3 Experimental dynamic structure factor. $S(k, E)$ determined by inelastic neutron scattering for a monolayer of liquid ${ }^{3} \mathrm{He}$ of areal density $4.7 \pm 0.2$ atoms $/ \mathrm{nm}^{2}$, shown as a function of the neutron momentum transfer $\hbar k$ and energy transfer $E$. The colour scale evolves from white to red (in arbitrary units proportional to neutron counts). White colour is also used in the lower part of the graph, where data cannot be exploited due to the large quasi-elastic background, and in the limits of low and high $k$ determined by the angular range covered by the detectors. The blue solid lines display the limits of the particle-hole band of a Fermi gas with the bare ${ }^{3} \mathrm{He}$ atomic mass. High intensity regions indicate the existence of modes with wave-vector $k$ and energy $E$, broadened by the experimental resolution. The zero-sound collective mode, visible around $k \sim 5 \mathrm{~nm}^{-1}$ and $E \sim 0.7 \mathrm{meV}$, is broadened inside the particle-hole band. It emerges beyond this band as a well-defined mode, displaying a minimum as a function of 
energy at $k=15.5 \mathrm{~nm}^{-1}$ and $E=0.4 \mathrm{meV}$. This fermionic collective mode closely resembles the phonon-maxon-roton dispersion relation of liquid ${ }^{4} \mathrm{He}$ (see Figure 1).

Figure 4 Theoretical dynamic structure factor. $S(k, E)$, calculated for a monolayer of liquid ${ }^{3} \mathrm{He}$ of areal density 4.9 atoms $/ \mathrm{nm}^{2}$, shown as a function of the wave-vector $k$ and energy $E$. At this areal density, the Fermi wave-vector is $k_{\mathrm{F}}=5.55 \mathrm{~nm}^{-1}$ and the Fermi energy $E_{\mathrm{F}}=$ $0.213 \mathrm{meV}$. The blue solid lines display the limits of the particle-hole band calculated for a Fermi gas with the bare ${ }^{3} \mathrm{He}$ atomic mass. High intensity regions indicate the existence of modes with wave-vector $k$ and energy $E$. The theoretical spectrum has been slightly broadened to make the sharp collective modes visible. The zero-sound collective mode, well defined at low wavevectors, enters the particle-hole continuum, is broadened, and finally emerges beyond the lower limit of the particle-hole band, displaying a minimum as a function of energy. A phononmaxon-roton type of dispersion relation is clearly seen.

Figure 5 Neutron spectra at selected wave-vectors. The spectra correspond to cuts at wavevectors 5.5, 12.5 and $16.5 \mathrm{~nm}^{-1}$ of the data shown in Figure 3. Crosses are the experimental data, with error bars calculated using the standard deviation of the neutrons counts; details on the statistical sample are given in the supplementary material). The red dashed lines are the results of RPA calculations (slightly broadened to make delta-functions visible). The results from our dynamic many-body theory are shown as green lines; blue lines denote theoretical results folded with the experimental resolution. The light blue area depicts the particle-hole band. Note that the broadening of the results of the dynamic theory is due to multi-pair excitations. 


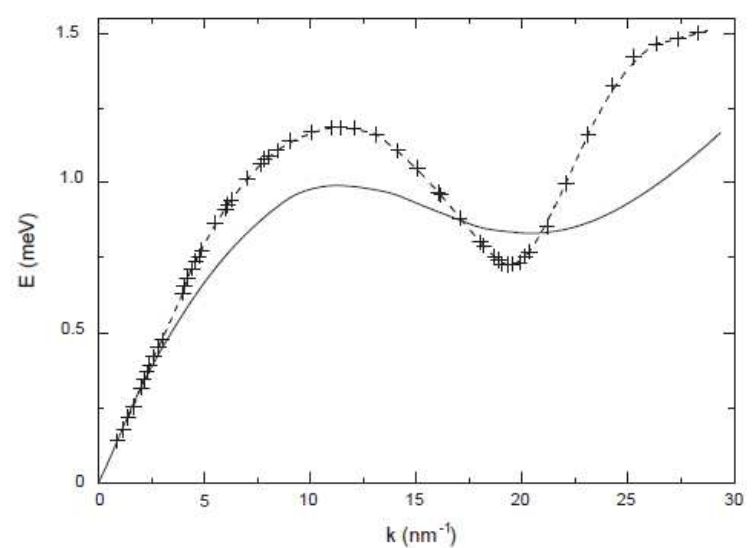

Figure 1

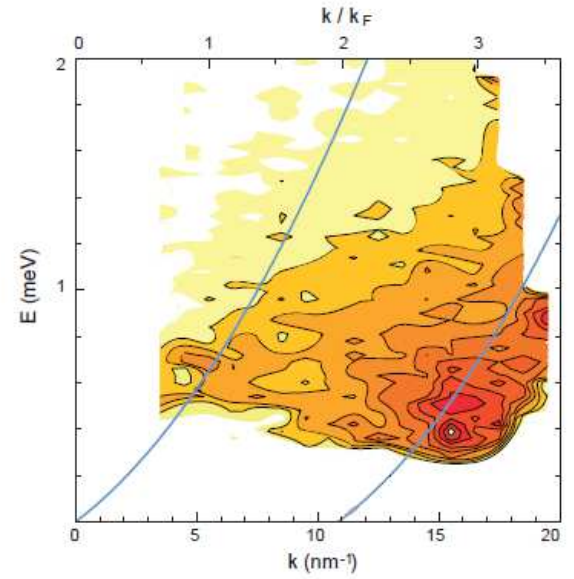

Figure 3

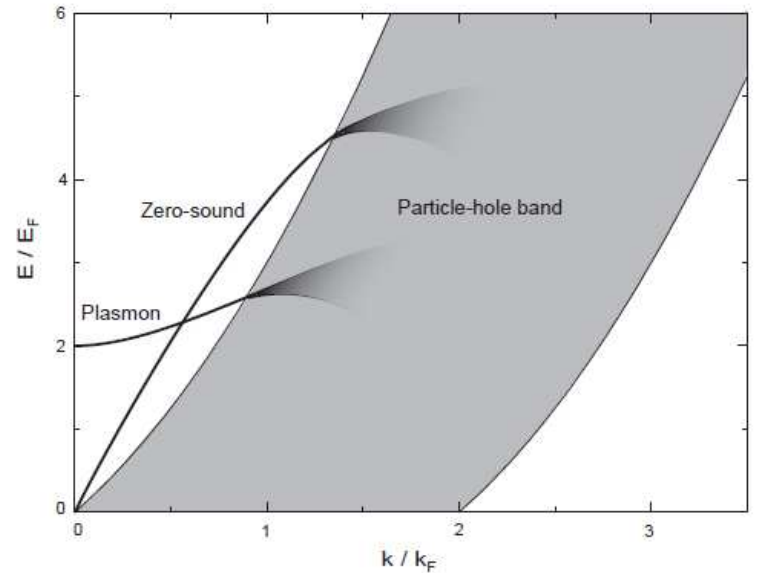

Figure 2

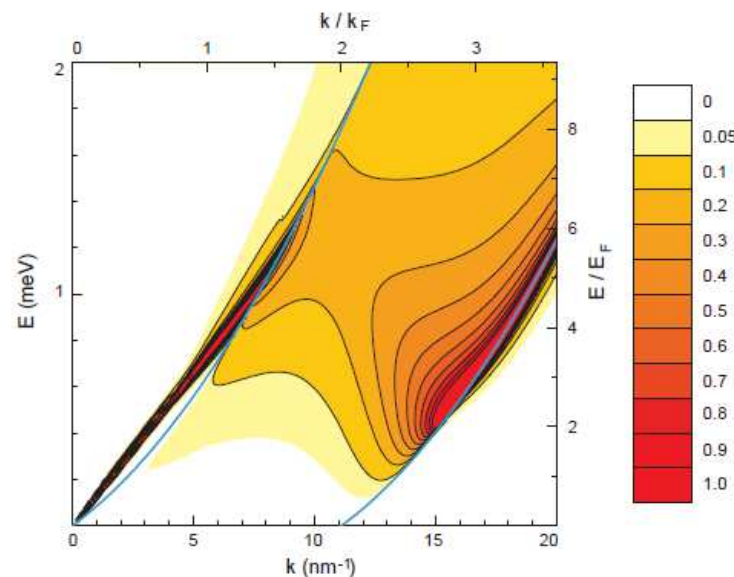

Figure 4
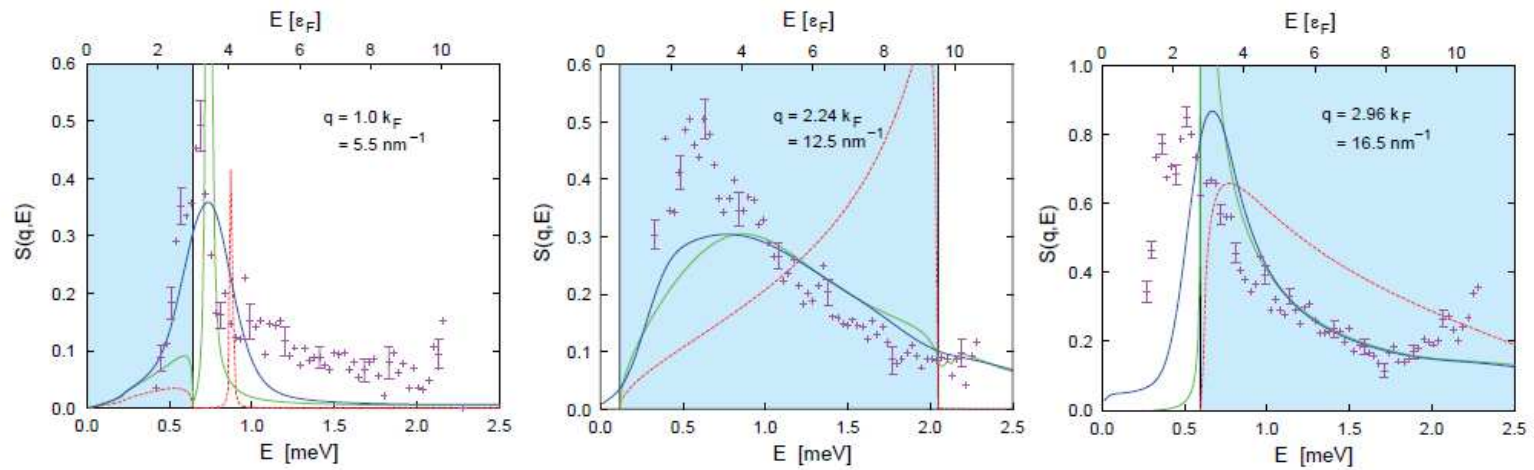

Figure 5 


\section{"Roton collective mode observed in a two-dimensional}

\section{Fermi liquid".}

\section{Supplementary material available on-line: experimental details}

\section{The ZYX exfoliated graphite sample}

The graphite substrate consists of a stack of high quality UCAR-ZYX (Union Carbide) exfoliated graphite plates of dimensions $25 \times 25 \times 5 \mathrm{~mm}^{3}$, with a total height of $6 \mathrm{~cm}$. The sample is larger than the beam size, defined by a rectangular neutron diaphragm of size $25 \times 50 \mathrm{~mm}$.

It is thermally anchored to the copper head of the aluminium sample cell by means of thin copper foils to the copper thermal connexion of experimental cell to the mixing chamber. The parts of the sample cell traversed by the neutron beam are made out of A5 aluminium.

Exfoliated graphite displays many interesting properties. In particular, this material offers large atomically flat platelets, a feature motivating their extensive use in the investigation of phase transitions in 2D adsorbed gases.

From an experimental point of view, exfoliated graphite is remarkably simple to clean: even exposed to air, it is only moderately contaminated, the loss of area is on the order of $5 \%$. A simple pumping procedure at moderate temperatures is sufficient to clean it, recovering completely the initial surface area. Therefore, accurate surface physics experiments can be performed without ultra-high vacuum conditions. In particular, water and hydrocarbons, the most frequent contaminants of porous materials, are not a problem in the case of exfoliated graphite.

The choice of the ZYX type of graphite substrate (compared for instance to Grafoil of Papyex exfoliated graphite) is dictated by several considerations:

a) The large size of the graphite platelets; the coherence length of ZYX $(190 \mathrm{~nm})$ ensures that size effects will not limit the life-time of the excitations.

b) The small mosaic spread (about 10 degrees), increases the signal collected by the detectors in the 2-dimensional in-plane geometry of the neutron scattering experiment. It also reduces the contamination by the (0002) reflection of the misaligned graphite crystallites.

c) The large graphite crystals ensure a good thermal conductivity, needed for low temperature experiments.

d) The neutron absorption due to a ${ }^{3} \mathrm{He}$ atomic layer, as described in the following sections, is optimised for the specific surface area of ZYX.

This sample has been used previously in several neutron scattering experiments on adsorbed ${ }^{3} \mathrm{He}$ and ${ }^{4} \mathrm{He}$ films. A complete set of neutron diffraction and inelastic scattering data for different neutron wave-lengths was therefore available. Hence, our sample is completely characterised; we show 
below how this valuable information was used to determine the density of the helium layers, and we provide the corresponding references to the literature.

\section{1) Graphite substrate area determination:}

The surface area of the graphite sample used in this experiment has been accurately determined by neutron diffraction and checked by adsorption isotherms at $4.20 \mathrm{~K}$.

The amount of gas corresponding to the perfect filling of the commensurate $\sqrt{3}$ phase of helium adsorbed on graphite, defined by the largest diffraction signal as a function of coverage, was measured as $14.351 \mathrm{~cm}^{3}$ of gas in standard conditions of temperature and pressure (STP: T=273.15 K, P=100 kPa).

Using $D_{2}$ adsorbed on the same sample we obtained by the same method $14.336 \mathrm{~cm}^{3}$ STP for the commensurate $\sqrt{3}$ phase, essentially the same value.

The amount of gas adsorbed on our ZYX graphite sample for a perfect filling of the commensurate $\sqrt{ } 3$ phase is taken as $V_{c}=14.34 \pm 0.02 \mathrm{~cm}^{3} S T P$.

Using the measured value of the lattice parameter of the commensurate phase (its density is found to agree with that inferred from the graphite lattice parameter and the geometry of the $\sqrt{3}$ phase), 6.366 atoms $/ \mathrm{nm}^{2}$, one can readily calculate the sample "commensurate area" $\mathrm{Ac}=\mathbf{5 9 . 7} \mathrm{m}^{2}$.

This value is useful as a reference, since the amount of gas needed to form the $\sqrt{ } 3$ commensurate phase can be determined by different methods (for instance by neutron diffraction, adsorption isotherms, heat capacity or NMR).

Near monolayer completion, due to the heterogeneity of the adsorption potential of the sample, the area available for adsorption increases with the total coverage, an effect which cannot be neglected. A diffraction measurement of the lattice parameter of the adsorbate is therefore performed in order to an accurate determine the density of the film as a function of coverage. The results are shown in Figure 1.

Using the known amount of adsorbed gas for several coverages in the incommensurate phase, below monolayer completion, one can readily calculate the so-called "incommensurate area" $A_{l}$ in the dense incommensurate solid phase. The corresponding effective area for adsorption at coverages around a complete monolayer is $A_{1}=65.3 \mathrm{~m}^{2}$.

In the presence of a partial second layer, a compression of the first layer is observed (see Figure 1). The neutron diffraction data allow determining the actual density of the first layer, the amount of ${ }^{4} \mathrm{He}$ adsorbed in the first layer; the number of atoms in the second layer is found as the difference between the total number of atoms introduced in the cell, and that in the compressed first layer. 


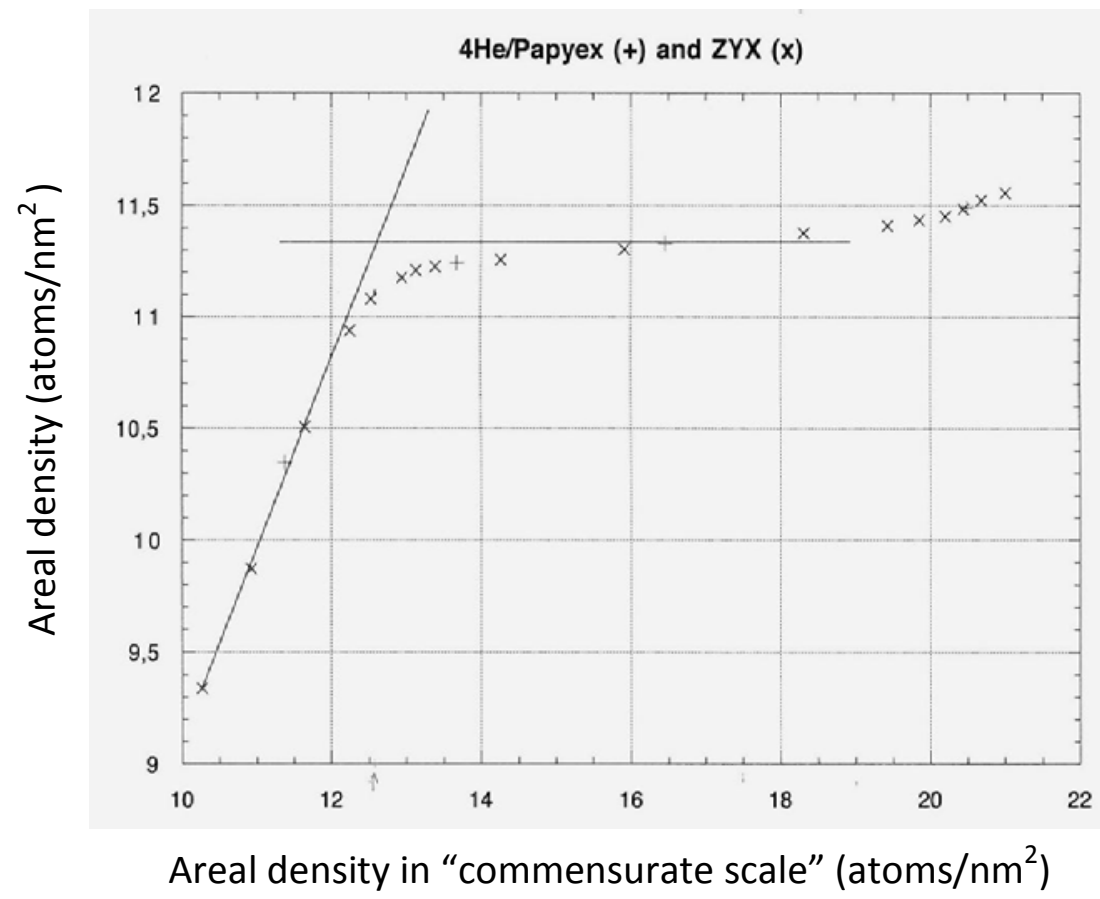

Figure 1: Density of ${ }^{4} \mathrm{He}$ layers adsorbed on two kinds of exfoliated graphite samples:

+ for Papyex; $x$ for ZYX (inferred from the lattice parameter directly measured by neutron diffraction), as a function of the commensurate scale density (i.e. the amount of adsorbed gas, normalised to the amount corresponding to the commensurate phase, times the density of the latter, 6.366 atoms $/ \mathrm{nm}^{2}$ ). This graph allows determining the distribution of the adsorbed atoms (amounts in the first and second layer, and their densities).

More details about exfoliated graphite, the calibration of areas, the definition of the commensurate and incommensurate areas, can be found in:

- Neutron diffraction studies of 2D quantum systems

H.J. Lauter, H.P. Schildberg, H. Godfrin, H. Wiechert, R. Haensel.

Canadian Journal of Physics, 65 (1987) 1435-1439.

- ${ }^{3}$ He layers on graphite

H.J. Lauter, H. Godfrin, V.L.P. Frank and H.P. Schildberg

Physica B 165\&166, 597-598 (1990).

- Experimental Properties of ${ }^{3} \mathrm{He}$ adsorbed on Graphite

H. Godfrin and H.J. Lauter

Progress in Low Temp. Physics, Vol. XIV, Chapter 4, p; 213-320, ed. by W.P. Halperin, Elsevier Science B.V., Amsterdam,1995.

- Systematic study of ${ }^{3} \mathrm{He}$ adsorbed on graphite by NMR techniques

C. Bäuerle, Y. Bunkov, S.N. Fisher and H. Godfrin

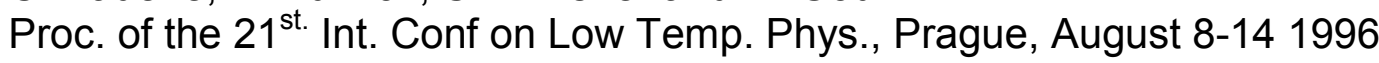

Czechoslovak J. of Phys. 46, suppl S1, 399, 1996 


\section{2) Density of the adsorbed helium layers}

The amount of ${ }^{4} \mathrm{He}$ introduced in the cell was $\mathbf{V}_{\mathbf{4}}=\mathbf{2 8 . 5 9} \mathbf{c m}^{3}$ STP. This is sufficient to complete the first monolayer considering the effect of the pressure of the partial second layer. The amount of ${ }^{3} \mathrm{He}$ was $\mathbf{V}_{3}=11.0 \mathrm{~cm}^{3}$ STP.

The total amount of gas introduced was therefore $V_{T}=39.59 \mathrm{~cm}^{3} S T P$. The first layer is pure ${ }^{4} \mathrm{He}$, and the second layer pure ${ }^{3} \mathrm{He}$, with a measured accuracy on the order of $1 \%$. This technique has been checked by NMR, a very sensitive tool to observe the promotion of ${ }^{3} \mathrm{He}$ atoms from a dense (solid) layer to a liquid overlayer:

- Two-dimensional Fermi-Liquid in the highly correlated regime: the second layer of ${ }^{3} \mathrm{He}$ adsorbed on graphite

K.-D. Morhard, C. Bäuerle, J. Bossy, Y. Bunkov, S.N. Fisher and H. Godfrin

Phys. Rev. B 53, 2658-2661 (1996).

- Ferromagnetic nanoclusters in two-dimensional ${ }^{3} \mathrm{He}$

E. Collin, C. Bäuerle, Yu. M. Bunkov and H. Godfrin

Phys. Rev. B 73, 125421 (2006)

The density of solid layers can be directly measured by neutron diffraction; this is not possible for the liquid: there is no sharp feature in its diffraction spectrum. The density of the second layer ${ }^{3} \mathrm{He}$ fluid can however be inferred from the amount of ${ }^{3} \mathrm{He}$ introduced, and the measured area of the sample. We do not know exactly the fraction of the substrate area explored by the ${ }^{3} \mathrm{He}$ atoms in the second layer, but the adsorption potential heterogeneity is small; the area covered by the liquid is larger than the "Commensurate area" $A_{c}$ of the sample, and smaller than its "incommensurate area" $A_{\mid}$. We expect that the fraction of the substrate area covered by the film to be in the centre of this range, since the liquid film investigated here is relatively dense, and its zeropoint motion large (comparable to that of bulk liquid ${ }^{3} \mathrm{He}$ at 10 Bars).

The density of the two-dimensional liquid ${ }^{3} \mathrm{He}$ film is found to be $\rho_{\mathrm{c}}=4.9$ atoms $/ \mathrm{nm}^{2}$ using the commensurate area, and $\rho_{1}=4.5$ atoms $/ \mathrm{nm}^{2}$ with the incommensurate area. This is the largest cause of uncertainty on the density, dominating all the error sources, including neutron diffraction, adsorption isotherms, temperature and pressure measurements used in the procedure.

The final result, using conservative estimates for the error bars, is that the liquid ${ }^{3} \mathrm{He}$ second layer density is $\rho=4.7 \pm 0.2$ atoms $/ \mathbf{n m}^{2}$. This accuracy is sufficient for the purposes of the present investigation, since all Fermi liquid properties are weakly dependent on density. 


\section{3) Background subtraction.}

The measurement of the dynamic structure factor of an atomic layer of ${ }^{3} \mathrm{He}$ is a particularly demanding experiment, for neutron scattering standards. The signal of a single atomic layer is small, and the neutron absorption cross section of the ${ }^{3} \mathrm{He}$ nucleus is huge. The graphite substrate, the copper thermal connection foils, the aluminium cell, the cryostat aluminium shields are in the neutron beam and contribute to the measured spectra as an intense background, energy and wave-vector dependent. Subtracting the background from the signal requires a delicate, long and tedious procedure:

a) First, a measurement of the signal is done at low temperatures ( $T<100 \mathrm{mK}$ ) with the bare graphite (without helium in the cell). The main characteristics of this background are:

- A large intensity is measured around $Q=0, E=0$, in the vicinity of the direct neutron beam ( $Q$ is the wave-vector, and $E$ the energy).

- An intense diffraction peak is measured at $Q=18.767 \mathrm{~nm}^{-1}$ corresponding to the (0002) reflection of the graphite. This Bragg peak has been studied for our sample in detail, performing rocking curves in order to determine the mosaic spread quoted earlier. The c-axis of the graphite is mainly oriented normal to the scattering plane, but due to the misalignment of the graphite platelets, a large Bragg peak is observed. Due to the finite energy resolution of the IN6 instrument $(\sim 0.1 \mathrm{meV})$, the Bragg peak wings contaminate the very low energy region of the spectrum. Using a graphite sample with a small mosaic spread is therefore a necessary condition for measuring inelastic spectra at low energies at wave-vectors on the order of $20 \mathrm{~nm}^{-1}$.

- As expected from the different sources of background, a large elastic signal is seen for all wave-vectors, spoiling the very low energy region of the spectra.

- A graphite phonon branch is observed, emerging from the graphite Bragg peak. This excitation is the only significant inelastic contribution in the background in the Q-E range of interest. Its width is limited in the present studies by the instrumental resolution of $0.1 \mathrm{meV}$. As discussed below, this feature is very helpful to check the accuracy of the background subtraction.

b) Second, a measurement is performed at low temperatures $(T<100 \mathrm{mK}$ ) with the graphite sample covered by the solid ${ }^{4} \mathrm{He}$ monolayer. The main observations under these conditions, are:

- The graphite Bragg peak becomes taller and narrower. This interference effect is due to the diffraction of graphite platelets of finite thickness; adding ${ }^{4} \mathrm{He}$ layers on both sides of the platelets leads to a reduction of the sizeeffect. This was demonstrated in several earlier studies of gases adsorbed on graphite (see references above).

No inelastic signals are seen in the region of interest. The density of the (triangular) solid ${ }^{4} \mathrm{He}$ layer is high, and the corresponding value of the Bragg wave-vector $\mathrm{Q}=22.6 \mathrm{~nm}^{-1}$ is beyond our range. At low wave-vectors, 
the solid ${ }^{4} \mathrm{He}$ phonons are not visible: their dispersion relation is much steeper than that of the liquid zero-sound mode, and the intensity is very small.

c) Third, a measurement is performed at low temperatures $(T<100 \mathrm{mK})$ with the graphite sample covered by the solid ${ }^{4} \mathrm{He}$ monolayer and the ${ }^{3} \mathrm{He}$ overlayer.

The graphite Bragg peak is further narrowed. Its amplitude, however, decreases markedly, due to the absorption of neutrons by the ${ }^{3} \mathrm{He}$ nuclei. The absorption correction factor is determined by computing the reduction of the integrated intensity around the elastic signal. The effect depends on the diffraction angle, due to the geometry of the sample. The signal is reduced by about $30 \%$ : this absorption is significant, but the signal is still large, and the correction is easy to apply.

d) In the $(Q, E)$ region accessible by the instrument IN6 (roughly $0<Q<20 \mathrm{~nm}^{-1}$; $0<E<2 \mathrm{meV}$ at the wavelength $\lambda=0.51 \mathrm{~nm}$ used here), our analysis must distinguish different regions:

- At small energies, the quasi-elastic background associated to the finite energy resolution of the instrument, is very large. It is still significant at "energies" on the order of 2 to 3 times the resolution. Since we are looking for very small signals, its subtraction from the signal leads to very large error bars. This region of the Q-E plane cannot be exploited. The effect is more pronounced at $\mathrm{Q} \sim 19 \mathrm{~nm}^{-1}$, due to the graphite Bragg peak.

- At small wave-vectors, the scattering is weak, leading to a reduced sensitivity. For this reason, for example, zero-sound is only visible at wavevectors larger than $3 \mathrm{~nm}^{-1}$.

- At higher wave-vectors and energies, the conditions for inelastic scattering studies are excellent: the background is small and does not display any structure, as observed in many experiments performed on the same sample and spectrometer.

e) For the reasons described above, the background subtracted from the data consists of the data obtained with the ${ }^{4} \mathrm{He}$ solid layer, corrected at low energies for the narrowing due to the additional ${ }^{3} \mathrm{He}$ layer. The correction procedure, based on smooth functional fits, does not introduce any feature in the Q,E plane that could be interpreted as an "excitation" (i.e. a peak in the $S(Q, E)$ function). By choosing different parameters for the background subtraction, we found that the procedure is reliable provided that $Q$ and $E$ are larger than some precise limits. In the contour plots shown in the article, the regions where the background cannot be subtracted reliably have been suppressed (white areas).

f) The measurements with the ${ }^{3} \mathrm{He}$ sample, in turn, are simply corrected for the neutron absorption, measured on the integrated graphite peak, using the measured attenuation factor. 
Typical raw spectra are shown below for the "substrate" (bare graphite covered by a monolayer of ${ }^{4} \mathrm{He}$, i.e. the background used for the present measurements), and for the ${ }^{3} \mathrm{He}$ sample adsorbed onto this substrate. Note the large variation in intensity between the different energy regions of the spectrum.

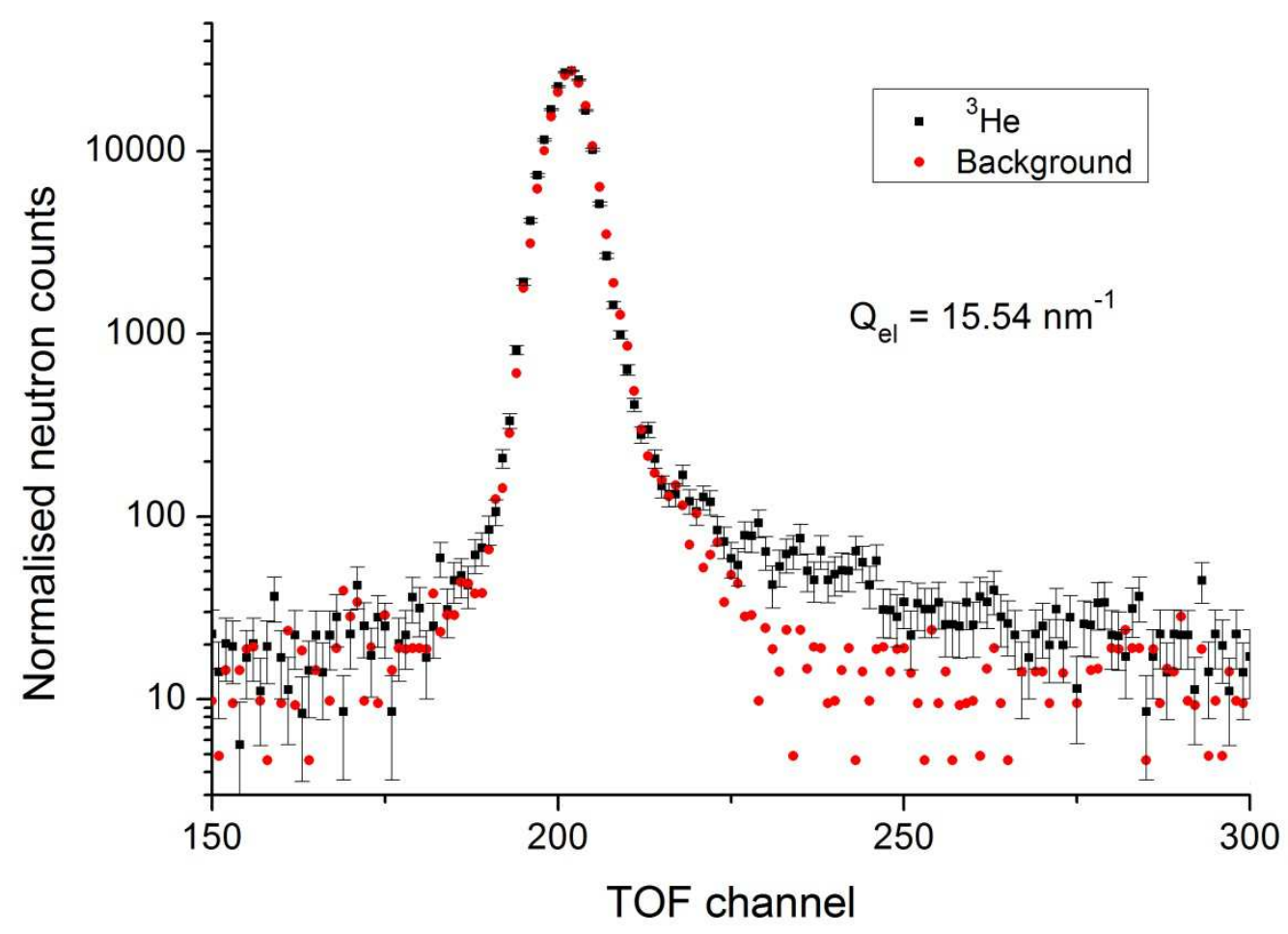

Time-of-Flight raw spectra taken at a diffraction angle of $78.33^{\circ}$ (the corresponding elastic wave-vector is $\mathrm{Q}=15.54 \mathrm{~nm}^{-1}$ ). A time-of-flight channel corresponds to $9.625 \mu \mathrm{sec}$.

The counts measured by 2 detectors out of 337 have been added. Note the logarithmic vertical scale (counts normalised to monitor and corrected for detector efficiencies). The data for ${ }^{3} \mathrm{He}$ have been corrected for the neutron absorption by a factor 1.35 , determined by scaling the elastic peaks. The actual number of neutron counts acquired in the experiment for our 23 hours scans is about one third of the indicated "normalised counts". The elastic peak is very intense, but relatively narrow for our ZYX graphite. Only a few neutrons per hour are collected in the region where the ${ }^{3} \mathrm{He}$ excitations are seen. Statistical error bars are shown only for the ${ }^{3} \mathrm{He}$ data for clarity. More than one hundred similar graphs have been used to generate the $S(q, \omega)$ contour plots shown in the article.

\section{4) Energy resolution of IN6.}

The resolution of the ILL neutron spectrometer IN6 is well known, since it has been used for far more demanding measurements in terms of energy and wave-vector resolution, namely the measurement of the dispersion relation of superfluid ${ }^{4} \mathrm{He}$ at very low temperatures.

See for instance: 
- TOF spectrometry: energy calibration and consistency check.

A. Stunault, K.H. Andersen, Y. Blanc, B. Fak, H. Godfrin, K. Guckelsberger and R. Scherm

Physica B 180\&181, 926-928 (1992).

- Collective excitations in liquid ${ }^{4} \mathrm{He}: \mathrm{I}$. Experiment and presentation of the data K.H. Andersen, W.G. Stirling, R. Scherm, A. Stunault, B. Fak, H. Godfrin and A.J. Dianoux

J. Phys.: Cond. Matter 6, 821-834 (1994).

- Precision determination of $S(Q, \Omega)$ of liquid ${ }^{4} \mathrm{He}$ as a function of temperature, K.H. Andersen, W.G. Stirling, R. Scherm, A. Stunault, B. Fak, H. Godfrin, A.J. Dianoux

Physica B 180\&181, 851-853 (1992).

The energy resolution for our wave-length of $0.51 \mathrm{~nm}$, is $0.08 \mathrm{meV}$ at low $\mathrm{Q}$, increasing with the wave-vector up to $0.12 \mathrm{meV}$.

\section{5) Width of the excitations observed in the $2 \mathrm{D}$ liquid ${ }^{3} \mathrm{He}$}

The spectrum of the liquid ${ }^{3} \mathrm{He}$ layer shown in the article on Figure 2a displays two main features: the broad, structure-less contribution of particle-hole excitations (particle-hole band, or PHB), and two regions corresponding to collective modes:

- At low wave-vector, the zero-sound mode. As seen on Figure 3 , at $k=5.5 \mathrm{~nm}^{-1}$, when the mode is at the edge of the PHB, a rather intense (for low- $Q$ standards) excitation is measured, in contrast with the broad distribution seen within the PHB, at $11.5 \mathrm{~nm}^{-1}$. Fitting with a Gaussian function the data for different values of the wave-vector (a rough procedure, mainly based on the high-energy side of the peak), we conclude that the zero-sound mode width is essentially described by the experimental energy resolution of $0.1 \mathrm{meV}$ (width $0.15 \pm 0.05 \mathrm{meV}$ ).

- At high wave-vectors, the re-emerging zero-sound mode is observed. The neutron peak is found to be just below the PHB, and hence this region of the spectrum contains contributions from the mode (broadened at least by the energy resolution) as well as contributions from the PHB. Fitting with a Gaussian the data for different values of the wave-vector (the fit is dominated by the low-energy side of the peak) we conclude that the reemerging zero-sound mode width is described well by the experimental energy resolution of $0.12 \mathrm{meV}$ (width $\sim 0.14 \pm 0.04 \mathrm{meV}$ ).

Since the measured width of the mode is at least limited by the experimental resolution, the actual width must be equal or smaller than $0.1 \pm 0.05 \mathrm{meV}$. It is worth pointing out that the Dynamic Many Body theory predicts a much smaller value, even though it includes the main relaxation processes leading to the finite life-time of this mode (multi-pair excitations, see Figure 3 ). 
We conclude that zero-sound emerges beyond the PHB as a well-defined excitation, its intrinsic line-width being significantly smaller than its energy, at least by a factor equal to three according to the experiment, and even more according to the theoretical calculation.

\section{6) Theory vs Experiment}

The experimentally determined $S(Q, \omega)$ of $2 D$ liquid ${ }^{3} \mathrm{He}$ is compared in this article to the result of the Dynamic Many-Body Theory (DMBT). We emphasize the striking similarity between theory and experiment in Figure 2. We also point out in Figure 3 the marked difference between former theoretical approaches, based on the Random Phase Approximation (RPA), and those provided by the DMBT. In particular, the spectral weight of the particle-hole excitation within the $\mathrm{PH}$ band is reversed.

Former experiments in bulk liquid ${ }^{3} \mathrm{He}$ as well as the present experiments could not be interpreted, even qualitatively, with the RPA results: theory and experiment were qualitatively different.

The agreement between the experiment and the DMBT results is therefore a remarkable event. On the one hand, it shows that taking into account dynamical correlations, it is possible to describe with a good accuracy the behavior of highly correlated fermionic systems. On the other hand, it constitutes an excellent tool in order to analyse the experimental data: as seen on Figure 3 , it is much easier to describe the spectrum in terms of DMBT, than in the framework of the RPA. This is valid for the collective excitations, the PH band, and also the region of multi-pairs, above the $\mathrm{PH}$ band.

The DMBT brings an excellent description of 2D liquid ${ }^{3} \mathrm{He}$, at an almost quantitative level. Differences between theory and experiment are still visible: in particular the roton-like minimum is observed experimentally below the particle-hole band. The DMBT succeeds in lowering the mode down to the lower edge of the band (RPA would locate this mode right in the middle of the $\mathrm{PH}$ band).

This effect is similar to that observed in the case of bosons: the roton energy is reduced as the theories become more accurate, starting from Feynman's theory, until present many-body calculations. The dynamic many-body theory of Jackson, Feenberg, and Campbell provides us now with an excellent description of the excitation spectrum of superfluid ${ }^{4} \mathrm{He}$, with a roton minimum a factor of two below Feynman's value.

Our present understanding of the DMBT indicates that higher order processes, involving more than 2 particle-hole pairs should reduce the roton-like energy.

Last but not least, it is worth pointing out that the theory and the experiments agree well not only on the nature of the excitations and their dispersion relation, but also on their intensity. The colour code used in Figures $2 a$ and $2 b$ emphasizes the remarkable agreement observed between the intensity of the experimentally determined $S(Q, \omega)$ and the DMBT calculation. This is verified in all the $Q, E$ plane explored here. 